\title{
PREFACE: INTERFACIAL DYNAMICS AND HEAT TRANSFER
}

Interfacial dynamics and heat transfer are encountered in a great number of industrial technologies; for example, engines, boilers, turbines, heat exchangers, condensers, fuel cells, microchip cooling systems, enhanced oil recovery technologies, etc. The research topics of interfacial dynamics and heat transfer have attracted continuous attention in multiple disciplines including thermal physics, fluid mechanics, and multiphase flows. To promote the development and optimization of those industrial technologies, understanding-from both fundamental and application viewpoints - of the fluid flows, heat and mass transfer, interfacial dynamics including interface deformation and morphology change, and their mutual couplings plays an extremely important role. However, there are still many challenges to fully understand these complex phenomena, since they often appear at multiscales, i.e., in macro-, meso-, micro-, and nano-scales. In addition, interfacial dynamics and heat transfer are usually involved with many other multiple physical and/or chemical processes simultaneously in industrial technologies, e.g., electrical field, magnetic field, acoustic field, chemical concentration field, chemical and electrochemical reactions, etc. It seems that interdisciplinary research using experimental, theoretical, and numerical approaches could be the right way to explore these complex phenomena and reveal their governing principles and mechanisms.

This special issue of Interfacial Dynamics and Heat Transfer is a collection of research papers involved in the interdisciplinary research area of interfacial phenomena, heat and mass transfer, and related multidisciplinary applications, in which several advanced research methods including theoretical, numerical, and experimental approaches are used. In the initial call of this issue, five specific topics are included: (1) nano- and micro-structured surfaces for enhancement of phase change phenomena; (2) contact line phenomena, droplets, spray, film flows, dry spots formation, wettability effect; (3) boiling, nucleation, bubbles, entrainment, critical heat flux; (4) thermocapillary flows, instability, interfacial wave, evaporation, condensation; and (5) two-phase flows, and heat and mass transfer in microchannels and ministructures.

All papers in this special issue have undergone a peer-review process to meet the high-quality requirements of the journal of Interfacial Phenomena and Heat Transfer. The Guest Editors would like to express our gratitude to all contributing authors and invited reviewers for their great efforts.

\section{Guest Editors:}

Hitoshi Asano

Department of Mechanical Engineering,

Kobe University,

Kobe, Japan

Irina Graur Martin

IUSTI Laboratory UMR CNRS 7343,

Aix Marseille University,

Marseille, France
Bo Feng Bai

State Key Lab of Multiphase Flow in Power Engineering, Xi'an Jiaotong University,

Xi'an, China

Hang Guo

College of Environmental and Energy Engineering,

Beijing University of Technology,

Beijing, China 